\title{
PONTLYFNI: A DIFFERENTIATED METEORITE RELATED TO THE GROUP IAB IRONS
}

\author{
ANDREW M. DAVIS, R. GANAPATHY * and LAWRENCE GROSSMAN ** \\ Department of the Geophysical Sciences, University of Chicago, Chicago, Ill. 60637 (USA)
}

Received December 13, 1976

Revised version received January 31,1977

\begin{abstract}
The abundances of 23 major and trace elements in the Pontlyfni meteorite have been measured by instrumental neutron activation analysis. The compositions of the metal and silicate fractions suggest a genetic relationship between Pontlyfni and the group IAB irons.
\end{abstract}

\section{Introduction}

The Pontlyfni meteorite fell on Coch-y-Bug Farm, Pontlyfni, Llanwnda, Caernarvonshire, Wales at 11:53; April 14, 1931 [1]. Most of the mass is believed to have fallen into the sea, but a 150 -g stone was seen to fall on land and was recovered. L.J. Spencer was allowed to examine the stone shortly after its fall, but he could only state that it was a meteorite of an unusual type. The meteorite was acquired for the British Museum by R. Hutchison in 1975 .

Graham et al. [2] have examined the specimen and confirmed that it is indeed an unusual meteorite. Their examination of Pontlyfni indicates that it is highly crystalline, containing forsterite and enstatite with smaller amounts of diopside and plagioclase. In addition, the meteorite contains about $25 \%$ metal and $21 \%$ sulfide by weight. A wet chemical analysis [2] shows that Pontlyfni has an oxidation state and total iron content close to those of enstatite chondrites, and $\mathrm{ag} / \mathrm{Si}$ ratio typical of ordinary chondrites. Graham et al. [2] propose that Pontlyfni is a member of a "K-chondrite" group, along with Kakangari $[3,4]$, Mount Morris (Wisconsin) and Winona. The so-called K-chondrites are characterized by $\mathrm{Mg} / \mathrm{Si}$ atomic ratios of $\sim 0.95$, large amounts of metal and sulfide, olivine

\footnotetext{
* Present address: J.T. Baker Chemical Company, 222 Red School Lane, Phillipsburg, N.J. 08865, U.S.A.

** Also Enrico Fermi Institute.
}

compositions of $\mathrm{Fa}_{1-5}$ and significant $\mathrm{Cr}$ in sulfides.

The oxygen isotopic composition of silicates from the Pontlyfni meteorite has been measured (R.N. Clayton, personal communication). On the three-isotope oxygen diagram, Pontlyfni lies slightly below the terrestrial mass fractionation line, identical in isotopic composition with silicates from the group IAB iron meteorites. Because of its unusual chemical and isotopic characteristics, we decided to determine the concentrations of a large number of trace and major elements in Pontlyfni in order to explore further its relationships with other meteorite types.

\section{Analytical methods and results}

We have analyzed a 34.1-mg piece of the Pontlyfni meteorite for 23 elements by intrumental neutron activation analysis using techniques described in previous publications from this laboratory $[5,6]$. The standard pot (SP $[7,8]$ ) was used as a standard for most elements. Their concentrations were taken from Perlman and Asaro [8]. The concentrations of Os, Ir and $\mathrm{Au}$ in Pontlyfni were determined from chemical standards which had been included in previous irradiations. The specific activities of ${ }^{191} \mathrm{Os},{ }^{192} \mathrm{Ir}$ and ${ }^{198} \mathrm{Au}$ at the end of the Pontlyfni irradiation were calculated from their values in previous irradiations by correcting for differences in neutron fluence using 
${ }^{46} \mathrm{Sc}$ in SP. The concentration of $\mathrm{Ca}$ in Pontlyfni was determined with the assumption that a $17 \cdot \mathrm{mg}$ sample of Kakangari, included in the same irradiation, has the same concentration as the Kakangari sample analysed by Mason and Wiik [9]. Lu was determined via the 208.4-keV $\gamma$-ray of ${ }^{177} \mathrm{Lu}$ which is interfered with by ${ }^{199} \mathrm{Au}$, having peaks at 158.4 and $208.2 \mathrm{keV}$. A ${ }^{199} \mathrm{Au}$ correction was not necessary for SP because no peak was observed at $158 \mathrm{keV}$. One was observed in the $\gamma$-ray spectrum of Pontlyfni, however, and the ${ }^{199} \mathrm{Au}$ contribution to the $208 \mathrm{-keV}$ peak was corrected for by using the $208-\mathrm{keV} / 158 \cdot \mathrm{keV}$ peak area ratio measured in a pure $\mathrm{Au}$ standard from a previous irradiation.

The results are shown in Table 1 , along with $1 \sigma$ standard errors based on counting statistics in sample and standard, and are compared with wet chemical data from Graham et al. [2]. Our value of $29.6 \%$ total $\mathrm{Fe}$ is lower than the value of $33.84 \%$ in Graham et al. [2], probably reflecting millimeter scale inhomogeneities in the distribution of metal and sulfide in Pontlyfni. When the two samples are corrected to the same

\section{TABLE 1}

Elemental abundances in Pontlyfni (in ppm, unless otherwise noted)

\begin{tabular}{|c|c|c|c|}
\hline \multicolumn{3}{|c|}{ This work } & \multirow{2}{*}{$\begin{array}{l}\text { Graham et al. [2] } \\
66003\end{array}$} \\
\hline $\mathrm{Na}$ & 6422 & \pm 7 & \\
\hline $\mathrm{Ca}$ & 1.18 & $\pm 0.07 \%$ & $0.99 \%$ \\
\hline $\mathrm{Sc}$ & 5.79 & \pm 0.01 & \\
\hline $\mathrm{Cr}$ & 2087 & \pm 6 & 2258 \\
\hline $\mathrm{Mn}$ & 2020 & \pm 30 & 1865 \\
\hline $\mathrm{Fe}$ & 29.6 & $\pm 0.2 \%$ & $33.84 \%$ \\
\hline Co & 883 & \pm 3 & 920 \\
\hline $\mathrm{Cu}$ & 185 & \pm 1 & \\
\hline $\mathrm{Zn}$ & 130 & \pm 4 & \\
\hline $\mathrm{Ga}$ & 16.6 & \pm 0.5 & \\
\hline As & 3.2 & \pm 0.1 & \\
\hline $\mathrm{Se}$ & 16 & \pm 9 & \\
\hline $\mathrm{Sb}$ & 0.39 & \pm 0.03 & \\
\hline $\mathbf{L a}$ & 0.26 & \pm 0.01 & \\
\hline $\mathrm{Ce}$ & 0.7 & \pm 0.2 & \\
\hline $\mathrm{Sm}$ & 0.103 & $3 \pm 0.007$ & \\
\hline $\mathbf{E u}$ & 0.10 & \pm 0.004 & \\
\hline $\mathrm{Yb}$ & 0.09 & \pm 0.02 & \\
\hline $\mathbf{L u}$ & 0.013 & $3 \pm 0.002$ & \\
\hline Hf & 0.11 & \pm 0.03 & \\
\hline Os & 0.78 & \pm 0.06 & \\
\hline Ir & 0.58 & $5 \pm 0.001$ & \\
\hline $\mathbf{A u}$ & 0.25 & \pm 0.002 & \\
\hline
\end{tabular}

proportions of metal, sulfide and silicate (see section 4), the concentrations of $\mathrm{Na}, \mathrm{Ca}, \mathrm{Cr}, \mathrm{Mn}$ and $\mathrm{Co}$ agree to within $10 \%$.

\section{Evidence of igneous differentiation in Pontlyfni}

Shown in Fig. 1 are the Si-normalized abundances of rare earth elements (REE) in Pontlyfni and Kakangari [4], divided by the Si-normalized abundances in $\mathrm{Cl}$ chondrites [10]. From the REE pattern, it is immediately obvious that Pontlyfni does not have the nearly unfractionated REE pattern typical of ordinary chondrites and Kakangari. It has a marked positive Eu anomaly and all other REE are depleted in it relative to chondritic abundances, with the heavier REE showing the larger depletions. From the fractionated REE pattern of Pontlyfni, it appears that igneous differentiation processes played a significant role in its evolution.

The ratios of some major and trace elements to $\mathrm{Si}$ in Pontlyfni are compared in Table 2 to those of Kakangari and two group IAB iron meteorites containing silicate inclusions, Campo del Cielo and Woodbine. The $\mathrm{Ca} / \mathrm{Al}$ atomic ratio found in Pontlyfni, 0.51 [2], is significantly lower than the remarkably constant ratios, $0.69-0.75$, found in all types of undifferentiated meteorites [11]. The fact that $\mathrm{Ca}$ and $\mathrm{Al}$ are very difficult to fractionate by condensation provides strong evidence for igneous differentiation processes in the origin of Pontlyfni.

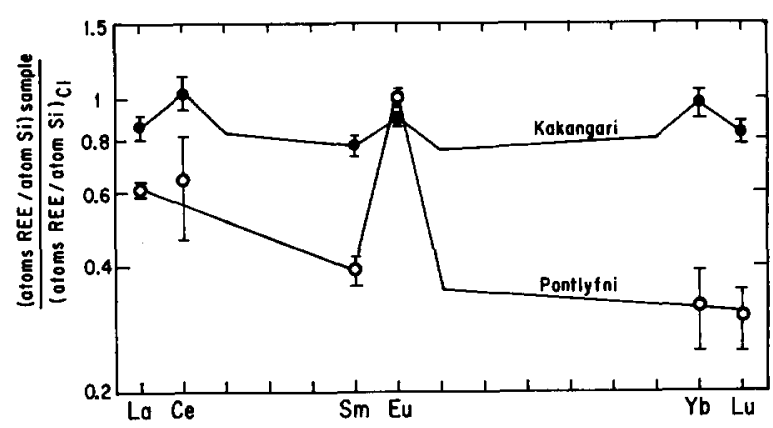

Fig. 1. Silicon-normalized atomic abundances of REE in Kakangari [4] and Pontlyfni (this work) divided by the silicon-normalized atomic abundances of REE in $\mathrm{Cl}$ chondrites [10]. 


\section{TABLE 2}

Major element atomic abundance ratios and silicate norms of Pontlyfni, Woodbine, Campo del Cielo and Kakangari

\begin{tabular}{|c|c|c|c|c|}
\hline & $\begin{array}{l}\text { Pontlyfni } \\
\text { [2] }\end{array}$ & $\begin{array}{l}\text { Woodbine } \\
\text { [19] }\end{array}$ & $\begin{array}{l}\text { e Campo } \\
\text { del Cielo } \\
{[12]}\end{array}$ & $\begin{array}{l}\text { Kakangari } \\
{[2]}\end{array}$ \\
\hline $\mathrm{Na} / \mathrm{Si}$ & 0.056 & 0.066 & 0.034 & 0.048 \\
\hline $\mathrm{Mg} / \mathrm{Si}$ & 0.95 & 0.90 & 1.07 & 0.98 \\
\hline $\mathrm{Al} / \mathrm{Si}$ & 0.094 & 0.073 & 0.060 & 0.071 \\
\hline $\mathrm{Ca} / \mathrm{Si}$ & 0.048 & 0.043 & 0.040 & 0.049 \\
\hline $\mathrm{Ca} / \mathrm{Al}$ & 0.51 & 0.58 & 0.67 & 0.70 \\
\hline $\mathrm{FeO} / \mathrm{Si}$ & 0.023 & 0.048 & 0.082 & 0.044 \\
\hline $\mathrm{Mn} / \mathrm{Si}$ & 0.007 & 0.003 & 0.007 & 0.008 \\
\hline Orthopyroxene & 51.46 & 46.75 & 44.97 & 49.19 \\
\hline Clinopyroxene & 4.81 & 7.51 & 3.54 & 5.13 \\
\hline Olivine & 25.67 & 28.86 & 40.07 & 30.21 \\
\hline Plagioclase & 17.29 & 16.52 & 10.36 & 13.84 \\
\hline Apatite & 0.30 & - & 0.55 & 0.85 \\
\hline Ilmenite & 0.23 & 0.18 & 0.20 & 0.19 \\
\hline Chromite & 0.24 & 0.18 & 0.30 & 0.58 \\
\hline
\end{tabular}

\section{Similarities and relationships to group IAB iron meteorites}

Pontlyfni and the silicate-bearing group IAB iron meteorites have similar mineralogical compositions. In addition to containing the same major and minor phases (enstatite, forsterite, plagioclase, diopside, nickel-iron, troilite, daubreelite and schreibersite), the compositions of pyroxene, olivine and plagioclase overlap. In Pontlyfni, orthopyroxene is $\mathrm{Fs}_{\mathbf{0 . 5 - 2 . 0}} \mathrm{Wo}_{1.6}$, olivine is $\mathrm{Fa}_{0.8-1.3}$, plagioclase is $\mathrm{Or}_{3} \mathrm{An}_{12}$ and clinopyroxene is $\mathrm{Fs}_{1.1} \mathrm{Wo}_{45.5}$ [2]. These mineral compositions are $\mathrm{Fs}_{4.0-8.7} \mathrm{Wo}_{1.1-2.1}, \mathrm{Fa}_{1-8}, \mathrm{Or}_{1.64 .1} \mathrm{An}_{9.2-21.5}$ and $\mathrm{Fs}_{1.9-3.9} \mathrm{Wo}_{41.8 .46 .4}$, respectively, in group IAB irons [12]. Although Graham et al. [2] do not include graphite among the minerals found in thin section, they did find $0.05 \% \mathrm{C}$ in the non-magnetic, acidinsoluble portion of their bulk analysis of Pontlyfni. The carbon in Pontlyfni may be present as graphite, a mineral which is found in all group IAB irons.

Bild [13] has shown that silicate from group IAB irons have variable REE patterns which are always strongly fractionated as is the case for Pontlyfni.

The oxygen isotopic composition of silicates from Pontlyfni suggests a relationship with the group IAB iron meteorites, and rules out genetic relationships with ordinary, enstatite and carbonaceous chondrites (R.N. Clayton, personal communication).

The sample of Pontlyfni analyzed by Graham et al. [2] contains $33.84 \%$ total $\mathrm{Fe}, 0.87 \% \mathrm{FeO}, 7.04 \% \mathrm{~S}$ and $2.19 \% \mathrm{Ni}$. Our sample of Pontlyfni contains $29.6 \%$ total Fe. If it is assumed that both samples have the same metal-to-sulfide ratio, the same concentration of $\mathrm{FeO}$ in the silicate fraction and the same metal composition, the weight percent metal in our sample can be calculated. The results of this calculation give $21.4 \%$ metal, $16.8 \%$ sulfide and $61.8 \%$ silicate. Assuming that the siderophile elements are totally concentrated in the metal phase, their abundances in it were calculated in order to compare them with the abundances found in the metal fraction of group IAB irons (Table 3). Carbon is considered a siderophile element in this computation because, for these concentrations, $\mathrm{C}$ is soluble in nickel-iron above $700^{\circ} \mathrm{C}$. The abundances of C [2], Co, Ni [2], Ga, As, Os, Ir and $\mathrm{Au}$ are within the ranges seen in the metal fraction of group IAB irons. Although the Ga content calculated for Pontlyfni metal, $78 \mathrm{ppm}$, falls within the range observed for group IAB, it is somewhat higher than the 50-60 ppm expected for a member of this group containing $9.15 \% \mathrm{Ni}$. As $\mathrm{Ga}$ exhibits significant lithophile tendencies in chondrites [14], our computation may overestimate the Ga content of the metal because some of the $\mathrm{Ga}$ in the bulk meteorite may reside in the silicate fraction. The similarity in metal phase compositions is another feature suggestive of a relationship between Pontlyfni and the group IAB irons.

Table 2 shows CIPW silicate norms calculated

\section{TABLE 3}

Siderophile element abundances in the metal fractions of Pontlyfni, group IAB irons and Kakangari (in ppm, unless otherwise indicated)

\begin{tabular}{llll}
\hline & Pontlyfni & IAB & Kakangari \\
\hline $\mathrm{C}$ & $2300[2]$ & $2600[19]$ & \\
$\mathrm{Co}$ & $4100^{*}$ & $4100-5200[20]$ & $2700[4]$ \\
$\mathrm{Ni}$ & $9.15 \%[2]$ & $5.9-14.5 \%[21]$ & $9.37 \%[2]$ \\
$\mathrm{Ga}$ & $78^{*}$ & $35-98[21]$ & $65[4]$ \\
$\mathrm{As}$ & $15^{*}$ & $5-19[20]$ & $11[4]$ \\
$\mathrm{Os}$ & $3.6^{*}$ & $2-5[20]$ & $7.6[4]$ \\
$\mathrm{Ir}$ & $2.7^{*}$ & $0.46-5.5[21]$ & $4.8[4]$ \\
$\mathrm{Au}$ & $1.17^{*}$ & $0.97-2.04[22,23]$ & $1.39[4]$ \\
\hline
\end{tabular}

* This work. 
from published bulk analyses of Pontlyfni, Woodbine, Campo del Cielo and Kakangari. That for Campo del Cielo was computed from the mean composition of three silicate inclusions [12] which differ substantially from one another in bulk chemistry (E. Jarosewich, personal communication). The silicate fraction of Pontlyfni has a very similar normative mineralogy to that of the group IAB iron Woodbine but differs significantly from that of Campo del Cielo, another group IAB iron. Insofar as the norm derived for Campo del Cielo is representative of the silicate fraction of the entire meteorite, it seems to be depleted in plagioclase and enriched in olivine relative to Pontlyfni and Woodbine. Such variations in normative mineralogy are easily accomplished during partial melting and fractional crystallization.

Most previous workers agree that the group IAB irons are products of igneous differentiation processses. The principal theories of origin of the group IAB irons are that they represent residues of primordial planetary melting and differentiation [15], old silicates taken up by a younger metal-sulfide melt [12], silicates accreted with large amounts of metal followed by some partial melting [13] or remnants of the coreforming process in which the molten-sulfide mixture was prematurely frozen $[16,17]$. The similarities between Pontlyfni and the group IAB irons suggest to us that they could have originated in the same igneous differentiation processes.

\section{Differences between Pontlyfni and Kakangari}

Kakangari and Pontlyfni resemble one another in olivine composition, $\mathrm{Mg} / \mathrm{Si}$ ratio and normative mineralogy of the silicate fraction. They both contain large amounts of metal and sulfide and significant amounts of $\mathrm{Cr}$ in their sulfides. For these reasons, Graham et al. [2] suggests that they are genetically related.

Kakangari and Pontlyfni differ in several fundamental respects, however. Kakangari fits the classical definition of a chondrite, containing numerous chondrules set in a fine-grained matrix [9], while Pontly fni has a fine-grained texture showing no chondrules or chondrule vestiges [2]. Kakangari has a flat REE pattern, with slight depletions of all REE relative to $\mathrm{Cl}$ chondritic abundances, while Pontly fni has a fractionated REE pattern with a strong positive $\mathrm{Eu}$ anomaly. The atomic $\mathrm{Ca} / \mathrm{Al}$ ratio of Kakangari, 0.70 [2], is within the range seen in ordinary chondrites, $0.69-0.75$ [11], while the Pontlyfni Ca/Al ratio is 0.51 [2], considerably lower than the chondritic value. The $\mathrm{C}$ content of Pontlyfni, $0.05 \%$ [2], also appears to be much lower than that of Kakangari, $0.20 \%$ according to Mason and Wiik [9] or $0.98 \%$ according to Graham et al. [2].

The abundances of trace elements in the metal, sulfide and silicate fractions of Kakangari and Pontlyfni also differ. The sample of Kakangari analyzed by Graham et al. [2] contains $22.79 \%$ total $\mathrm{Fe}, 1.89 \%$ $\mathrm{FeO}, 5.30 \% \mathrm{~S}$ and $1.39 \% \mathrm{Ni}$, while the sample of Kakangari analyzed by Davis et al. [4] contains $21.1 \%$ total Fe. The calculated amounts of metal, sulfide and silicate in the Kakangari sample analyzed by Davis et al. [4] are $14.8,14.8$ and $70.4 \mathrm{wt} . \%$, respectively. The abundances of the siderophile elements $\mathrm{Co}, \mathrm{Ni}, \mathrm{Ga}, \mathrm{As}, \mathrm{Os}, \mathrm{Ir}$ and $\mathrm{Au}$ calculated for the metal fractions of Pontlyfni and Kakangari are compared in Table 3. It can be seen that while the abundances of most siderophile elements in the metal fraction are similar in the two meteorites, Kakangari's is substantially lower in Co. The abundances of trace elements in the sulfide and silicate fractions are compared in Table 4. The sulfide fractions of the two meteorites have similar amounts of $\mathrm{Cu}$, but Kakangari is $50 \%$ higher in $\mathrm{Zn}$ and $\mathrm{Se}$ and $50 \%$ lower in Sb than Pontlyfni Our heretofore unpublished value for the Se content of Kakangari is $21 \pm 12 \mathrm{ppm}$. The large uncertainties in the absolute Se contents of both Pontlyfni (Table 1) and Kakangari are due to poor counting statistics in the irradiation standard. In spite of this, the relative Se contents in the two meteorites are known to within $2 \%$. The silicate fraction of Kakangari is lower in $\mathrm{Ca}$ and higher in Sc, Hf and all REE except $\mathrm{Eu}$ than is that of Pontlyfni. On the three-isotope oxygen diagram, Kakangari plots on an extension of the $\mathrm{C} 2$ matrix mass fractionation line [18], well below the terrestrial mass fractionation line, while Pontlyfni and silicates from the group IAB irons plot only slightly below the terrestrial mass fractionation line (R.N. Clayton, personal communication). The Kakangari and Pontlyfni points can be joined by a line of slope $\sim 2$. The proposal by Graham et al. [2] that there is a genetic relationship between Kakangari and Pontlyfni must invoke an isotopic mixing process between rather unlikely end-members. 
TABLE 4

Inferred compositions of the sulfide and silicate fractions of Pontlyfni and Kakangari (values in ppm, unless otherwise indicated)

\begin{tabular}{ccc} 
& Pontlyfni & Kakangari \\
\hline Sulfide fraction & $16.8 \% *$ & $14.8 \% *$ \\
$\mathrm{Cu}$ & 1100 & 1150 \\
$\mathrm{Zn}$ & 770 & 1160 \\
$\mathrm{Sb}$ & 2.3 & 1.2 \\
$\mathrm{Se}$ & 94 & 141 \\
& & \\
Silicate fraction & $61.8 \% *$ & $70.4 \% *$ \\
$\mathrm{Na}$ & $1.04 \%$ & $0.99 \%$ \\
$\mathrm{Ca}$ & $1.91 \%$ & $1.68 \%$ \\
$\mathrm{Se}$ & 9.37 & 12.36 \\
$\mathrm{Hf}$ & 0.18 & 0.20 \\
$\mathrm{La}$ & 0.42 & 0.51 \\
$\mathrm{Ce}$ & 1.13 & 1.56 \\
$\mathrm{Sm}$ & 0.167 & 0.281 \\
$\mathrm{Eu}$ & 0.162 & 0.125 \\
Yb & 0.15 & 0.38 \\
$\mathrm{Lu}$ & 0.021 & 0.050 \\
\hline
\end{tabular}

* Entries refer to the abundances of the silicate and sulfide fractions, in weight percent of the bulk meteorite.

Any model proposing a genetic relationship between Kakangari and Pontlyfni faces severe constraints both from the differing abundances of major and trace elements and from the differing amounts of the ${ }^{16} \mathrm{O}$-rich component.

\section{Summary}

The similarities in oxidation state of the silicate fractions and trace element compositions of the metal fractions, coupled with the presence of fractionated rare earth patterns suggest a genetic relationship between Pontlyfni and the group IAB irons. The oxygen isotopic composition and the mineralogy are also consistent with such a relationship. These similarities suggest that they may have been involved in the same igneous differentiation processes. Although certain similarities in composition do exist between Pontlyfni and Kakangari, differences in $\mathrm{Ca} / \mathrm{Al}$ ratios, major and trace element abundances and oxygen isotopic compositions argue strongly against a genetic relationship.
We suggest that Pontlyfni is not a highly crystalline chondrite, but rather an achondrite closely related to the group IAB iron meteorites.

\section{Acknowledgements}

The sample of Pontlyfni was generously provided by R. Hutchison. We thank R. Hutchison, R.N. Clayton and R.W. Bild for permission to cite unpublished data and E. Anders, R.W. Bild, R. Hutchison and E. Jarosewich for valuable discussions. This research was supported by funds from the Alfred P. Sloan Foundation and by the National Aeronautics and Space Administration through grant NGR 14-001249.

\section{References}

1 A. King, The meteorite of 1931, April 14, J. Brit. Astron. Assoc. 42 (1932) 328-332.

2 A.L. Graham, A.J. Easton and R. Hutchison, Forsterite chondrites - the meteorites Kakangari, Mount Morris (Wisconsin), Pontlyfni and Winoma, submitted to Min. Mag. (1977).

3 A.L. Graham and R. Hutchison, Is Kakangari a unique chondrite?, Nature 251 (1973) 128-129.

4 A.M. Davis, L. Grossman and R. Ganapathy, Yes, Kakangari is a unique chondrite, Nature (1977) in press.

5 L. Grossman and R. Ganapathy, Volatile elements in Allende inclusions, Proc. 6th Lunar Sci. Conf., Geochim. Cosmochim. Acta, Suppl. 6, 2 (1975) 1729-1736.

6 L. Grossman and R. Ganapathy, Trace elements in the Allende meteorite, I. Coarse-grained, Ca-rich inclusions, Geochim. Cosmochim. Acta 40 (1976) 331-344.

7 I. Perlman and F. Asaro, Pottery analysis by neutron activation, Archaeometry 11 (1969) $21-52$.

8 I. Perlman and F. Asaro, Pottery analysis by neutron activation, in: R.H. Brill. ed., Science and Archaeology, (M.I.T. Press, Cambridge, Mass., 1971) 182-195.

9 B. Mason and H.B. Wiik, The composition of the Bali, Frankfort, Kakangari, Rose City and Tadjera meteorites, Am. Mus. Novit. 2272 (1966).

10 N. Nakamura, Determination of REE, Ba, Fe, Mg, $\mathrm{Na}$ and $\mathrm{K}$ in carbonaceous and ordinary chondrites, Geochim. Cosmochim. Acta 38 (1974) 757-775.

11 L.H. Ahrens, The composition of stony meteorites, IX. Abundance trends of the refractory elements in chondrites, basaltic achondrites and Apollo 11 fines, Earth Planet. Sci. Lett. 10 (1970) 1-6.

12 T.E. Bunch, K. Keil and E. Olsen, Mineralogy and petrology of silicate inclusions in iron meteorites, 
Contrib. Mineral. Petrol. 25 (1970) 297-340.

13 R.W. Bild, A study of primitive and unusual meteorites, Ph.D. Thesis, Univ. of California, Los Angeles, Calif. (1976).

14 C.L. Chou, P.A. Baedecker and J.T. Wasson, Distribution of $\mathrm{Ni}, \mathrm{Ga}, \mathrm{Ge}$ and Ir between metal and silicate portions of H-group chondrites, Geochim. Cosmochim. Acta 37 (1973) 2159-2171.

15 D.S. Burnett and G.J. Wasserburg, ${ }^{87} \mathrm{Rb}-{ }^{87} \mathrm{Sr}$ ages of silicate inclusions in iron meteorites, Earth Planet. Sci. Lett. 2 (1967) 397-408.

16 W.R. Kelly and J.W. Larimer, Chemical fractionations in meteorites, VIII. Iron meteorites and the cosmochemical history of the metal phase, Geochim. Cosmochim. Acta 41 (1977) 93-111.

17 E.R. Rambaldi and J.W. Larimer, The Shaw chondrite, I. The case of the missing metal, Earth Planet. Sci. Lett. 33 (1976) $61-66$.
18 R.N. Clayton, N. Onuma and T.K. Mayeda, A classification of meteorites based on oxygen isotopes, Earth Planet. Sci. Lett. 30 (1976) 10-18.

19 B. Mason, The Woodbine meteorite, with notes on silicates in iron meteorites, Mineral. Mag. 36 (1967) 120-126.

20 E.R.D. Scott, Chemical fractionation in iron meteorites and its interpretation, Geochim. Cosmochim. Acta 36 (1972) 1205-1236.

21 J.T. Wasson, Meteorites (Springer-Verlag, New York, N.Y., 1974).

22 E. Goldberg, A. Uchiyama and H. Brown, The distribution of nickel, cobalt, gallium, palladium and gold in iron meteorites, Geochim. Cosmochim. Acta 2 (1951) 1-25.

23 K.F. Fouché and A.A. Smales, The distribution of gold and rhenium in iron meteorites, Chem. Geol. 1 (1966) $329-339$. 